\title{
FATORES DE RISCO ASSOCIADOS À DEPRESSÃO GERIÁTRICA: REVISÃO INTEGRATIVA DA LITERATURA
}

\section{RISK FACTORS ASSOCIATED WITH GERIATRIC DEPRESSION: INTEGRATIVE LITERATURE REVIEW}

\author{
Ana Caroliny Oliveira da Silva ${ }^{1} *$ Gabriela Duarte Bezerra ${ }^{2} *$ Marcia Eduarda Nascimento dos $^{6}$ \\ Santos $^{3} *$ Micaelle de Sousa Silva $^{4} *$ Natannael da Silva Pereira ${ }^{5} *$ Vaneska Hellen Campos Araruna $^{6} *$ \\ Vinícius Alves de Alencar Oliveira ${ }^{7}$ Aline Sampaio Rolim de Sena ${ }^{8}$ \\ Vitória Ferreira Marinho ${ }^{9}$ * Rosely Leyliane dos Santos ${ }^{10}$
}

\begin{abstract}
RESUMO
Identificar os fatores de risco para a depressão em pessoas idosas. Trata-se de uma revisão integrativa da literatura, realizada no mês de dezembro de 2020, por meio do Portal de Periódicos da CAPES e da base de dados MEDLINE, via plataforma PUBMED e BVS. Os critérios de inclusão foram artigos publicados entre 2015 a 2020, completos e disponíveis na íntegra, nos idiomas inglês, português e espanhol. Por conseguinte, teses, dissertações, estudos de caso, capítulos de livros e artigos duplicados foram excluídos deste estudo. A amostra final foi composta por 11 artigos. Constatou-se que os fatores de riscos para a depressão em idosos incluem baixo nível de escolaridade, estado civil divorciado, solteiro ou viúvo, presença de mais de uma patologia crônica, ausência de atividades sociais, de lazer e de práticas de atividades físicas. O conhecimento acerca dos fatores de risco pode auxiliar na prevenção do desenvolvimento da depressão nesta população. Destaca-se, portanto, a importância de buscar compreender e investigar os fatores que influenciam esse transtorno, a fim de direcionar ações de saúde para a promoção da qualidade de vida dos idosos.
\end{abstract}

Palavras-chave: Assistência a Idosos. Depressão; Fatores de Risco

\section{ABSTRACT}

Identify risk factors for depression in the elderly. This is an integrative literature review, carried out in December 2020, through the CAPES Journal Portal and the MEDLINE database, via the PUBMED and VHL platform. The inclusion criteria were articles published between 2015 and 2020, complete and available in full, in English, Portuguese and Spanish. Therefore, duplicated theses, dissertations, case studies, book chapters and articles were excluded from this study. The final sample consisted of 11 articles. It was found that the risk factors for depression in the elderly include low education level, divorced, single or widowed marital status, presence of more than one chronic pathology, absence of social activities, leisure and physical activities. Knowledge about risk factors can help prevent the development of depression in this population. Therefore, the importance of seeking to understand and investigate the factors that influence this disorder is highlighted, in order to direct health actions to promote the quality of life of the elderly.

Keywords: Old Age Assistance; Depression; Risk Factors.

\footnotetext{
${ }^{1}$ Graduanda em Enfermagem. Universidade Regional do Cariri - URCA. Crato, Ceará, Brasil. E-mail: caroliny.oliveira@urca.br. Orcid: https://orcid.org/0000-0003-2457-2663

${ }^{2}$ Graduanda em Enfermagem. Universidade Regional Do Cariri - URCA. Crato, Ceará, Brasil. E-mail: gabriela.duarte@urca.br. Orcid: http://orcid.org/0000-0002-7472-4621

${ }^{3}$ Graduanda em Enfermagem. Universidade Regional do Cariri- URCA, Crato, Ceará, Brasil E-mail: eduardamaviael99@gmail.com. Orcid: https://orcid.org/0000-0002-0035-1754

${ }^{4}$ Graduanda em Enfermagem pela Universidade Regional do Cariri- URCA, Crato, Ceará, Brasil. E-mail: micaelle.sousa@urca.br. Orcid https://orcid.org/0000-0001-8729-8919

${ }^{5}$ Graduando em Enfermagem pela Universidade Regional do Cariri-URCA. Crato, Ceará, Brasil. E-mail: natannael.silva@urca.br. Orcid: https://orcid.org/0000-0002-6894-6439

${ }^{6}$ Graduando em Enfermagem. Universidade Regional do Cariri - URCA. Crato, Ceará, Brasil. E-mail:

vaneskahellen098@gmail.com. Orcid: https://orcid.org/0000-0003-3061-2756

${ }^{7}$ Graduando em Enfermagem. Universidade Regional do Cariri - URCA. Crato, Ceará, Brasil. Email: Vinícius.enfoliveira@ urca.br. Orcid: https://orcid.org/0000-0001-5602-0623

${ }^{8}$ Universidade Regional do Cariri-URCA. Crato, Ceará, Brasil. E-mail: aline_senna2008@ hotmail.com. Orcid: http://orcid.org/00000002-7819-4170

${ }^{9}$ Graduanda em Enfermagem. Universidade Regional do Cariri- URCA. Crato, Ceará, Brasil. E-mail: vitoria.marinho@urca.br. Orcid: https://orcid.org/0000-0002-4013-1019

${ }^{10}$ Doutora em Enfermagem. Docente da Universidade Regional do Cariri- URCA. Crato, Ceará, Brasil. Email: rosely.enfa@yahoo.com.br. Orcid: https://orcid.org/0000-0002-3908-8834
} 


\section{INTRODUÇÃO}

A depressão é um tipo de transtorno de humor considerado fenômeno complexo e multidimensional, que envolve fatores genéticos, biológicos, ambientais e psicológicos; podendo afetar diretamente a pessoa em sua saúde mental, convivência social e qualidade de vida. Com isso, estas circunstâncias podem interferir na capacidade do indivíduo em trabalhar, dormir, alimentarse e pode acarretar isolamento social ${ }^{(1-2)}$.

A depressão se destaca por sua alta prevalência e alta morbidade que pode ser caracterizada em leve, moderada ou grave a depender da intensidade dos sintomas. Os principais sintomas relacionados a este transtorno são: humor deprimido, anedonia, mudança no padrão de sono, cansaço, dificuldade para pensar, desesperanças e ideias suicidas. Esses sintomas, se não tratados de forma adequada, podem se tornar crônicos ou reincidentes ${ }^{(1-3)}$.

O diagnóstico da doença é feito pela investigação da sintomatologia, intensidade e duração, como também pela história clínica do paciente. O diagnóstico diferencial é importante pois a depressão pode ser sintoma secundário de outras doenças ${ }^{(4)}$.

O Brasil é considerado um país ansioso com a população que mais sofre de depressão da América Latina. De acordo com dados da Organização Mundial da Saúde (OMS), no país, cerca de 5,8\% da população possuem a depressão, afetando 11,5 milhões de brasileiros. Estes números foram crescentes no ano de 2020 em decorrência da pandemia provocada pelo Novo Coronavírus, em que houve um aumento de $68 \%$ de quadros depressivos no Brasil ${ }^{(5-6)}$.

A taxa de prevalência desse transtorno em pessoas acima de 60 anos, torna-se fator preocupante na sociedade brasileira, em que cerca de 28 milhões de pessoas encontram-se nesta faixa etária, representando $13 \%$ da população do país ${ }^{(7)}$. De acordo com Fernandes e colaboradores ${ }^{(8)}$, o sexo mais acometido por este tipo de transtorno é o feminino, em virtudes de fatores biológicos, genéticos e hormonais como também, as situações de conflitos pessoais de maior predominância neste gênero.

Apesar desta problemática ser prevalente, o número de casos diagnosticados de depressão, em pessoas idosas, é reduzido. Acredita-se que 50\% dos casos de depressão em idosos não sejam diagnosticados, devido a semelhança de outros sintomas naturais decorrentes do processo de envelhecimento com a doença. Alguns dos sintomas presente nesta faixa etária, que contribui para a confusão de diagnósticos são: queixas físicas, como a fadiga, sono, falta de apetite, indisposição; que podem ser confundidos pelo processo de adaptação do envelhecimento ${ }^{(9)}$.

Assim, tendo em vista o ritmo do envelhecimento na população mundial, que se 
estima um aumento de $80 \%$ da população idosa em 2050, o sub-tratamento e subdiagnóstico, nesta população, é importante conhecer os fatores relacionados e que poderão contribuir para o aumento de consequências como a vulnerabilidade social e também, o crescente número à ideação suicida $^{(10-11)}$.

Com isso, torna-se importante a investigação e compreensão dos fatores de risco relacionados ao desenvolvimento da depressão em idosos para que este transtorno não seja confundido com processos naturais da idade. No intuito de contribuir para o conhecimento dos profissionais da saúde, especialmente para a enfermagem, por ser uma profissão que atua e implementa melhorias na assistência em saúde à população idosa; este estudo é relevante. Assim, objetivou-se identificar os fatores de risco para a depressão em idosos.

\section{MÉTODO}

Trata-se de uma revisão integrativa. Para a construção deste estudo foram seguidas

Quadro 1: Elaboração da pergunta norteadora por meio da estratégia PVO. Crato, CE, Brasil, 2020.

\begin{tabular}{|l|l|l|l|}
\hline Elementos do método & Componentes & $\begin{array}{l}\text { Descritores do } \\
\text { assunto (DeCS) }\end{array}$ & $\begin{array}{l}\text { Descritores } \\
\text { assunto (MeSH) }\end{array}$ \\
\hline População & Pessoas Idosas & Assistência a Idosos & Old Age Assistance \\
\hline Variável & Fatores de Risco & Fatores de Risco & Risk Factors \\
\hline
\end{tabular}


Resultados

Diagnóstico

de Depressão

Depression

Depressão

Elaborado pelos autores, 2020.

Posteriormente, definiram-se os Descritores em Ciências da Saúde (DeCs) e os Medical Subject Headings (MeSh). Os DeCS e MeSh empregados foram os seguintes: Assistência a idosos/ Old Age Assistance, Depressão/Depression e Fatores de Risco/Risk Factors. Esses termos foram cruzados entre si por meio da utilização do operador booleano "AND”.

Nesse sentido, a coleta de dados foi realizada no mês de dezembro de 2020, a partir do Portal de Periódicos da Coordenação de Aperfeiçoamento de Pessoal de Nível Superior (CAPES) e na base de dados Medical Analysus And Retrieval System Online (MEDLINE) através da plataforma National Library of Medicine (PUBMED) e Biblioteca Virtual em Saúde (BVS).

Definiram-se como critérios de inclusão os artigos que correspondiam ao período de 2015 a 2020, com o objetivo de obter dados atuais da literatura. Além disso, levou-se em consideração estudos primários, que estivessem com texto completo e disponíveis na íntegra gratuitamente, nos idiomas inglês, português e espanhol.
Por conseguinte, teses, dissertações, capítulos de livros, estudos de casos, revisões de literatura e artigos duplicados foram excluídos deste estudo. Após a leitura dos resumos, os estudos que não relacionavam depressão a idosos e/ou fatores de risco foram excluídos.

Identificaram-se um total de 2.782 artigos, sendo que 77 foram encontrados no Portal de Periódicos Capes, 1.913 na BVS e 792 na PubMed. Posteriormente, 2.494 artigos foram excluídos após a análise do título e resumo, restando 288 para leitura na íntegra. Destes, 11 estudos foram incluídos com a aplicação dos critérios de exclusão e inclusão.

$\mathrm{O}$ instrumento Preferred Reporting Items for Systematic Rewiew and MetaAnalyses (PRISMA) ilustra o processo de busca e seleção dos estudos, conforme o Quadro 2. 
Quadro 2. Fluxograma relacionado ao processo de busca e seleção dos estudos. Crato, CE, Brasil, 2020.
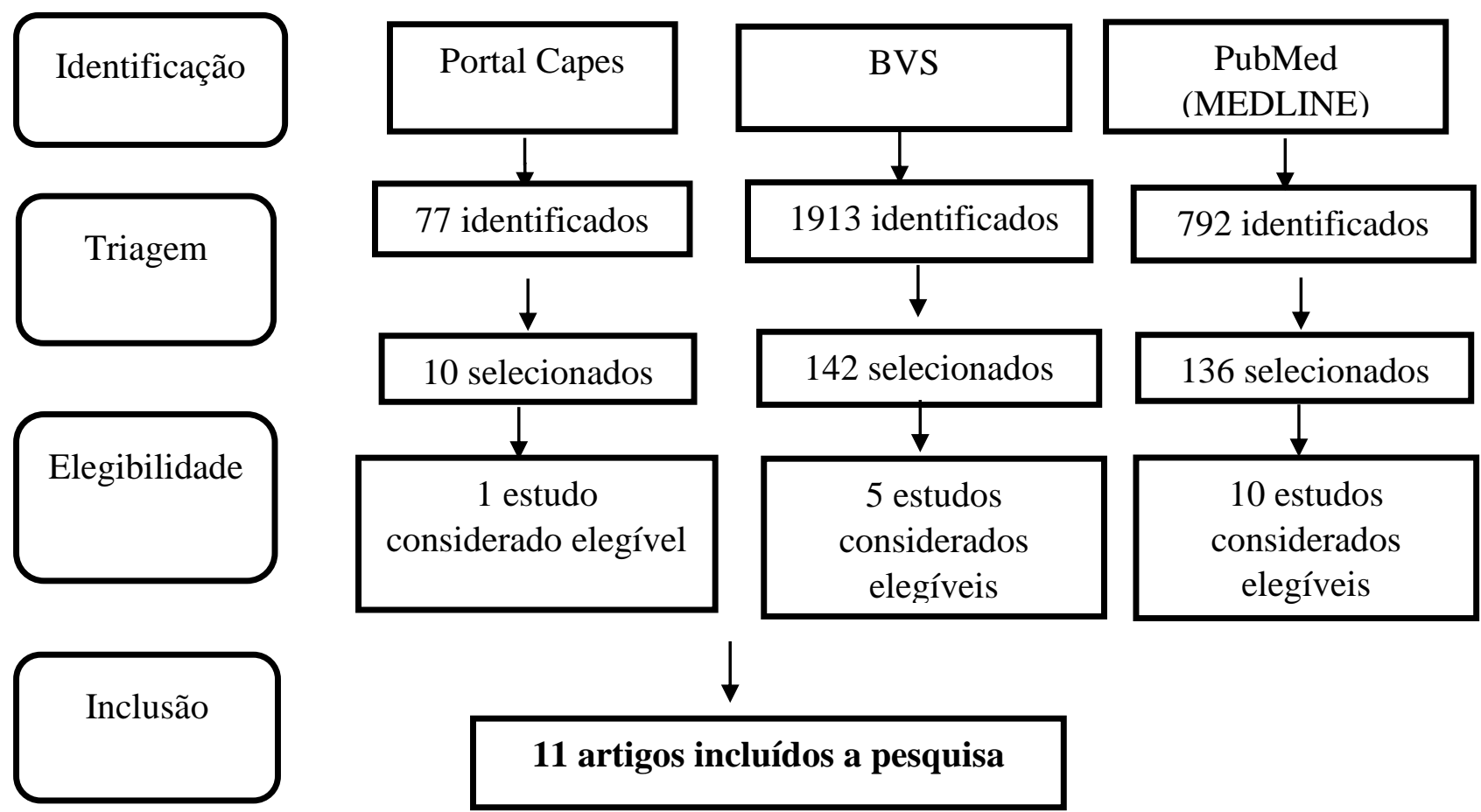

Elaborado pelos autores, 2020.

Efetuou-se a categorização dos estudos que compuseram a amostra de acordo com a classificação de qualidade das evidências, classificada em VII níveis: Níveis de Evidência (NE) I, as evidências são provenientes de revisão sistemática ou metanálise de ensaios clínicos randomizados controlados ou oriundos de diretrizes clínicas baseadas em revisões sistemáticas de ensaios clínicos randomizados controlados; NE II, evidências derivadas de pelo menos um ensaio clínico randomizado controlado bem delineado; NE III, evidências obtidas de ensaios clínicos bem delineados sem randomização; NE IV, evidências provenientes de estudos de coorte e de caso controle bem delineados; NE V, evidências originárias de revisão sistemática de estudos descritivos e qualitativos; NE VI, evidências derivadas de um único estudo descritivo ou qualitativo; NE VII, evidências oriundas de opinião de autoridades e/ou relatório de comitês de especialistas ${ }^{(13)}$.

Após a amostra final dos estudos incluídos, houve a interpretação e síntese dos artigos. Desse modo, os achados foram organizados de forma ordenada, sistemática e abrangente, sendo organizados por ano de publicação, tipo de estudo, título do artigo, autores, objetivo e nível de evidência. 


\section{RESULTADOS}

A amostra desta revisão foi composta por onze estudos que abordam os fatores associados à depressão em idosos. Desses, 1 pertencia ao Portal de Periódicos Capes, 5 a BVS e 5 a PubMed. Dentre eles, quatro foram publicados na língua portuguesa, um no idioma espanhol e o restante na língua inglesa, entre o período de 2015 e 2020, sendo predominante as publicações no ano de 2019.

Em relação aos periódicos nos quais foram publicados os artigos incluídos, quatro faziam parte de revistas de medicina, três de revistas de psiquiatria geriátrica, dois de revistas de epidemiologia e os demais, de outras revistas. As pesquisas desenvolveramse, predominantemente, em Unidades Básicas de Saúde (UBS) e sob a forma de inquérito domiciliar ou hospitalar.

Quanto aos instrumentos de pesquisa utilizados nos estudos que compõe esta revisão, a Escala de Depressão Geriátrica (EDG) foi aplicada em todos os casos, assim como questionários sobre o perfil sócio demográfico. Além disso, em alguns estudos foram utilizados questionários estruturados que abordavam Atividades de Vida Diária de Barthel (ADL), Atividades Instrumentais da Vida Diária (IADL), condições e uso de serviços de saúde e Questionário Internacional de Atividade Física (IPAQ).

Os artigos selecionados, bem como as informações referentes aos mesmos foram identificados a partir do Quadro 3, com as principais informações que caracterizavam de forma metodológica as publicações incluídas.

Quadro 3 - Apresentação da síntese de artigos incluídos na revisão integrativa. Crato, CE, Brasil, 2020.

\begin{tabular}{|c|c|c|c|c|c|}
\hline ANO & $\begin{array}{l}\text { TIPO DE } \\
\text { ESTUDO }\end{array}$ & $\begin{array}{l}\text { TITULO DO } \\
\text { ARTIGO }\end{array}$ & AUTORES & OBJETIVO & $\begin{array}{l}\text { NIVEL } \\
\text { DE } \\
\text { EVIDÊE } \\
\text { NCIA } \\
\text { (NE) }\end{array}$ \\
\hline 2015 & $\begin{array}{l}\text { Estudo cruzado } \\
\text { de avaliação } \\
\text { geriátrica } \\
\text { abrangente. }\end{array}$ & $\begin{array}{|lr|}\text { Riesgo } & \text { de } \\
\text { depresiónen } & \text { personas } \\
\text { de } 75 \text { anõs o } & \text { más, } \\
\text { valoración } & \text { geriátrica } \\
\text { integral y factores de } \\
\text { vulnerabilidadasociad } \\
\text { osen r Atención } \\
\text { Primaria. }\end{array}$ & Rubiano et al. & $\begin{array}{l}\text { Conhecer a prevalência de } \\
\text { risco de depressão, } \\
\text { principais características e } \\
\text { fatores vulnerabilidade } \\
\text { geriátrica associada. }\end{array}$ & IV \\
\hline
\end{tabular}




\begin{tabular}{|c|c|c|c|c|c|}
\hline 2018 & $\begin{array}{l}\text { Estudo } \\
\text { transversal }\end{array}$ & $\begin{array}{l}\text { A study on prevalence } \\
\text { of depression and } \\
\text { associate drisk factor } \\
\text { samongel derly in a } \\
\text { rural block of Tamil } \\
\text { Nadu }\end{array}$ & $\begin{array}{l}\text { BuvneshkumAR } \\
\text { et al. }\end{array}$ & $\begin{array}{l}\text { Estimar a prevalência de } \\
\text { depressão em idosos e } \\
\text { avaliar os fatores que estão } \\
\text { associados à depressão em } \\
\text { idosos. }\end{array}$ & $\overline{\mathrm{VI}}$ \\
\hline 2018 & $\begin{array}{l}\text { Estudo } \\
\text { transversal } \\
\text { longitudinal. }\end{array}$ & \begin{tabular}{|l} 
Sintomas depressivos \\
em idosos do \\
município de São \\
Paulo, \\
prevalência e fatores \\
associados \\
SABE)
\end{tabular} & Chiloff et al. & $\begin{array}{l}\text { Estimar a prevalência de } \\
\text { sintomas depressivos em } \\
\text { idosos do município de São } \\
\text { Paulo, Brasil (Estudo } \\
\text { SABE), em 2006 e } \\
\text { identificar fatores de risco } \\
\text { associados a essa } \\
\text { prevalência em } 2006 \text { e } \\
\text { fatores de proteção entre os } \\
\text { idosos que não } \\
\text { apresentaram } \\
\text { depressivos nas avaliações } \\
\text { realizadas em } 2000 \text { e } 2006 .\end{array}$ & $\overline{\mathrm{VI}}$ \\
\hline 2018 & $\begin{array}{l}\text { Estudo } \\
\text { qualitativo }\end{array}$ & $\begin{array}{l}\text { Fatores Associados à } \\
\text { Sintomatologia } \\
\text { Depressiva em Idosos }\end{array}$ & Lampert et al. & $\begin{array}{l}\text { Identificar a ocorrência de } \\
\text { sintomas depressivos na } \\
\text { população idosa e os fatores } \\
\text { envolvidos. }\end{array}$ & VI \\
\hline 2018 & $\begin{array}{l}\text { Estudo } \\
\text { transversal }\end{array}$ & $\begin{array}{l}\text { Prevalence and } \\
\text { associated factors of } \\
\text { depressive } \\
\text { symptom } \\
\text { samongelderly in } \\
\text { patients of a Chinese } \\
\text { tertiary hospital. }\end{array}$ & Zou et al. & $\begin{array}{l}\text { Avaliar a prevalência de } \\
\text { sintomas depressivos e } \\
\text { fatores associados entre } \\
\text { idosos e pacientes } \\
\text { internados. }\end{array}$ & VI \\
\hline 2019 & $\begin{array}{l}\text { Estudo } \\
\text { comparativo } \\
\text { transversal }\end{array}$ & \begin{tabular}{|l} 
Comparative \\
assessmentof \\
psychosocial status \\
ofelderly in urbanand \\
rural areas, \\
Karnataka, India
\end{tabular} & Akila et al. & $\begin{array}{l}\text { Fornecer uma avaliação } \\
\text { comparativa do estado } \\
\text { psicológico e social dos } \\
\text { idosos que vivem em áreas } \\
\text { urbanas e rurais de } \\
\text { Karnataka e identificar } \\
\text { fatores associados à } \\
\text { ocorrência de problemas } \\
\text { psicológicos em idosos. }\end{array}$ & VI \\
\hline 2019 & $\begin{array}{ll}\text { Estudo } & \\
\text { transversal de } & \\
\text { base } & \\
\text { populacional } & \end{array}$ & $\begin{array}{ll}\text { Prevalence } & \text { of } \\
\text { geriatric } & \text { depression in } \\
\text { the } & \text { Kavredistrict, } \\
\text { Nepal: } & \text { Findingsfrom } \\
\text { a cross } & \\
\text { sectionalcommunitysu } \\
\text { rvey. }\end{array}$ & Manandhar et al. & $\begin{array}{l}\text { Estimar a prevalência de } \\
\text { depressão entre os idosos } \\
\text { no distrito de Kavre. }\end{array}$ & $\overline{\mathrm{VI}}$ \\
\hline
\end{tabular}




\begin{tabular}{|c|c|c|c|c|c|}
\hline 2019 & $\begin{array}{l}\text { Pesquisa } \\
\text { Multicêntrica }\end{array}$ & $\begin{array}{l}\text { Depressive Symptoms } \\
\text { in Elderly Chinese } \\
\text { Primary Care } \\
\text { Patients: Prevalence } \\
\text { and } \\
\text { Sociodemographic } \\
\text { and Clinical } \\
\text { Correlates. }\end{array}$ & Zhong et al. & $\begin{array}{l}\text { Estimar a prevalência de } \\
\text { sintomas depressivos e } \\
\text { identificar as características } \\
\text { sociodemográficas } \\
\text { correlatos clínicos de } \\
\text { depressão em uma amostra } \\
\text { de pacientes idosos tratados } \\
\text { no ambiente de atenção } \\
\text { primária em Wuhan, China. }\end{array}$ & IV \\
\hline 2019 & $\begin{array}{l}\text { Estudo } \\
\text { transversal }\end{array}$ & $\begin{array}{l}\text { Fatores intervenientes } \\
\text { nos indicativos de } \\
\text { depressão } \\
\text { em idosos usuários } \\
\text { das unidades básicas } \\
\text { de saúde de } \\
\text { Maringá, Paraná, } \\
\text { 2017. }\end{array}$ & Oliveira et al & $\begin{array}{l}\text { Analisar os fatores } \\
\text { intervenientes nos } \\
\text { indicativos de depressão em } \\
\text { idosos das unidades básicas } \\
\text { de saúde (UBS) do } \\
\text { município de Maringá, } \\
\text { Paraná, Brasil, em } 2017 \text {. }\end{array}$ & VI \\
\hline 2020 & $\begin{array}{l}\text { Estudo } \\
\text { transversal }\end{array}$ & $\begin{array}{l}\text { Depression among } \\
\text { elderly users of } \\
\text { open and closed care } \\
\text { facilities in a } \\
\text { rural region of greece: } \\
\text { anImportant } \\
\text { public health issue. }\end{array}$ & Kouvatsou et al. & \begin{tabular}{lrr} 
Explorar & \multicolumn{2}{r}{ possíveis } \\
correlações & entre as \\
variáveis & \multicolumn{2}{r}{ acima } \\
mencionadas, como um \\
todo.
\end{tabular} & VI \\
\hline 2020 & $\begin{array}{l}\text { Estudo } \\
\text { transversal }\end{array}$ & $\begin{array}{l}\text { Screening of } \\
\text { undiagnosed } \\
\text { depression } \\
\text { amongelderly primary } \\
\text { carepatients: a cross- } \\
\text { sectional study } \\
\text { fromthe Republicof } \\
\text { Srpska, Bosniaand } \\
\text { Herzegovina. }\end{array}$ & Stanetié et al & $\begin{array}{lrr}\begin{array}{l}\text { Examinar a } \\
\text { depressão }\end{array} & \text { não } \\
\text { diagnosticada } & \text { entre } \\
\text { pacientes } & \text { idosos } & \text { de } \\
\text { cuidados primários } & \text { na } \\
\text { entidade da } & \text { República } & \text { de } \\
\text { Srpska } & \text { (Bósnia } & \text { e } \\
\text { Herzegovina), bem como os } \\
\text { fatores } & \text { de } & \text { risco } \\
\text { sociodemográficos } & \text { e } \\
\text { clínicos associados } & \text { à } \\
\text { depressão. } & \end{array}$ & VI \\
\hline
\end{tabular}

Elaborado pelos autores, 2020.

Constatou-se que a maioria dos estudos testou a associação de sintomas depressivos com diversos tipos de variáveis. Dessa forma, os fatores de risco encontrados foram agrupados em cinco dimensões: fatores de riscos sociodemográficos; capacidade funcional prejudicada; suporte social e eventos de vida

estressores; condições de saúde e nível de prática de atividade física e de lazer.

\section{Fatores de riscos sociodemográficos:}

Dentre sóciodemográficos, os elementos que apresentaram forte influência com a depressão em pessoas idosas estão relacionados a situação conjugal, nível de escolaridade, 
situação financeira e condições de moradia (21).

A situação conjugal manifestou relação com os sintomas depressivos em três estudos. Nesse quesito, em idosos divorciados, solteiros ou viúvos; apresentaram mais sintomas depressivos quando comparados com os casados (14-15-16). Além disso, baixo nível de escolaridade também foi associado com depressão em quatro artigos. Onde entende-se, que, quanto maior o nível educacional, menor o risco de eventos depressivos ${ }^{(14-17,18,19)}$. Nos demais estudos, a associação de escolaridade com depressão não foi percebida.

A auto percepção de situação financeira ruim, assim como a dependência financeira, foram vinculadas à depressão geriátrica em cinco estudos (14,15-17-19,20). Relacionado a condições de moradia, de acordo com estudo ${ }^{(17)}$, idosos com maior risco de desenvolver depressão são os residentes de áreas rurais, quando comparados a moradores de áreas urbanas, fator de risco indicado por mais um estudo ${ }^{(18)}$. Outro fator de risco citado relacionado a ocorrência de depressão em idosos, foi a condição de moradia. Idosos que viviam em casas de apoio ${ }^{(16)}$ e idosos que moravam sozinhos ${ }^{(21)}$, eram mais propensos ao desenvolvimento da depressão.

\section{Capacidade funcional prejudicada:}

Quando se trata da capacidade funcional, a incapacidade ou limitação funcional, evidenciadas por dependência para realização das atividades básicas de vida diária, foram significativamente associados à sintomatologia depressiva nos idosos em dois $\operatorname{artigos}{ }^{(14-22)}$.

\section{Suporte social e eventos de vida estressores:}

Quanto ao suporte social, estudos alertam como potenciais fatores de risco não ter ninguém a quem confiar quando se tem um problema (14-17-23), tempo limitado de convívio com a família e exposição verbal e/ou abuso físico ${ }^{(18-19-24)}$. No que se refere aos eventos estressores de vida, a vivência de situações que envolvem conflitos na família, morte de um membro da família ou pessoa próxima, doença de si mesmo/membro da família e situação de desemprego, foram relacionadas ao risco de depressão em idosos (15-22).

\section{Condições de saúde:}

As condições de saúde, tanto físicas quanto psíquicas, também implicam diretamente no risco de idosos desenvolverem depressão. Assim, a presença de mais de uma patologia crônica (doença cardíaca, deficiência visual, artrite, anemia, dor crônica, hipertensão arterial e câncer) foram consideravelmente associadas a incidência de depressão em seis estudos (14-15-16-19-20-23). Avaliar o estado geral de saúde, como ruim 
também se mostrou aspecto de risco para depressão ${ }^{(20-22)}$.

Dessa forma, questões que envolviam histórico de morbidades psicológicas, como deficiência mental percebida ${ }^{(17),}$ histórico de depressão anterior ${ }^{(14)}$ e aumento na escala de ansiedade ${ }^{(23)}$ foram citados como potenciais para desenvolvimento da depressão geriátrica. Além disso, dois artigos citaram o uso de mais dois medicamentos regularmente ${ }^{(14-20)}$, outros dois enfatizaram o declínio cognitivo (21-24) e, o histórico de quedas (20) como componentes de risco para os sintomas depressivos.

\section{Nível de prática de atividade física e de lazer:}

Quando se trata de nível de prática de atividade física e de lazer, três estudos revelaram a ausência das mesmas ${ }^{(19-20-23)}$ e dois a falta de atividades sociais e de lazer ${ }^{(16-}$ 23), como elementos fortemente associados a depressão geriátrica.

\section{DISCUSSÃO}

Perante os resultados obtidos, compreende-se que os fatores de risco inseridos no grupo abordado impactam fortemente na saúde mental dos idosos, especialmente quando se trata da depressão, por percorrer aspectos psicológicos, físicos, emocionais, sociais e comportamentais ${ }^{(25)}$.

Dessa forma, idosos com os fatores de risco apresentados estão vulneráveis, não apenas a depressão, como também a dificuldades de acesso aos serviços de saúde, ausência de apoio integral na prevenção da doença, especialmente, por disfunção na tríade indivíduo-família-comunidade. Pode haver ainda, dificuldades das equipes de saúde em prestar cuidados holísticos ao lidar com as especificidades das dinâmicas familiares ou mesmo com o próprio idoso, fator que compromete a resolutividade da assistência em saúde. Esse apoio institucional é relevante, sobretudo, no cuidado dos idosos com limitações físicas e mentais ${ }^{(26)}$.

Uma abordagem com 42 idosos residentes em uma unidade filantrópica na Bahia, buscava verificar a prevalência e os fatores associados a sintomas depressivos. Foi apontado que os fatores relacionados à depressão, nesses idosos institucionalizados, eram a insatisfação de viver com o desconhecido e seguir uma rotina de horários, perda da autonomia e poder de escolha somado ao fato de não se sentirem importantes ${ }^{(27)}$.

Estudo com pessoas de idade acima de 65 anos, na Espanha, constatou que 12,6\% da população estudada apresentava transtornos depressivos. Destes, 16, 8\% eram mulheres e $7,1 \%$ homens. Ainda houve diferença entre homens e mulheres referentes a não saber ler/escrever; presença de doença crônica e a 
falta de interesse das outras pessoas, gerando um sentimento de solidão ${ }^{(28)}$.

Esses dados implicam diretamente em pontos preponderantes da saúde mental dos idosos, sendo o público feminino com maior destaque para a propensão ao desenvolvimento da depressão, possivelmente por terem uma trajetória de vida com sobrecargas fisiológicas, emocionais e psicológicas de maior peso, determinando assim fatores necessários para maiores ações de integralidade na saúde.

Outrossim, também foram indicados como preditores para sintomas depressivos: vida assistida, ensino médio ou abaixo desse nível educacional, deficiência de membros superiores (DASH $\geq 50 \%$ ), sexo feminino e função cognitiva prejudicada (MEEM <26). Assim, é possível observar que a demência, causadora de prejuízo na função cognitiva está entre um dos principais distúrbios neuropsiquiátricos que afetam a saúde mental dos idosos e que podem gerar incapacidade $(30,31)$.

Essa condição é comumente associada aos idosos que possuem interação social apenas dependente de família. Em contrapartida, aqueles que possuem uma rede de apoio social mais integrada, apresentaram menores índices de desenvolvimento da demência. Sendo assim, uma relação equilibrada entre apoio familiar e um nível adequado de interação com amigos e/ou vizinhos para mantê-los envolvidos com o meio social, ajudaria a retardar tanto o desenvolvimento da demência quanto da depressão, já que ambas possuem uma associação entre si e a depressão também está intimamente ligada com o nível de engajamento social do idoso ${ }^{(31,30)}$.

Os dados asseguram a importância de saber identificar fatores de riscos ao desenvolvimento da depressão e para além, em casos de distúrbios neuropsíquicos, a demência vem sendo retratada como inimigo comum para idosos que vivem em situações socioeconômicas difíceis e sem apoio familiar, sendo assim um componente satisfatório para uma maior cobertura de cuidados evitando assim surgimentos de complicações futuras e possibilitando uma melhora na qualidade de vida e bem estar deste indivíduo.

Fatores importantes na melhora do estilo de vida, podendo prevenir ou retardar o declínio cognitivo associado ao envelhecimento são as atividades físicas. A implementação de atividades como aumento de 100 passos / dia na atividade física dos participantes, resultou na probabilidade de manifestação de redução em 14\% de chances de desenvolverem a depressão, segundo a Escala de Depressão de Goldberg ${ }^{(32,33)}$.

Assim, a atividade física pode ser considerada um fator que diminui o estado de depressão, ansiedade, distúrbios do sono, dor 
e mal humor. Fato esse que elucida a correlação entre o índice de depressão em pessoas idosas que vivem em casas de repouso com as atividades que possam ser realizadas fora deste ambiente, já que quanto maior o nível de atividade física, mais estes residentes irão dispor de condições para visitar lugares diferentes e interagir com outras pessoas, viabilizando um maior estado de saúde mental ${ }^{(33,34) \text {. }}$

Dessa forma, é essencial adotar medidas para um envelhecimento ativo e prevenção da depressão, que envolve a integração de aspectos individuais, econômicos, sociais, físicos, comportamentais, de serviços sociais e de saúde. Assim, são necessárias intervenções preventivas para a população idosa, por meio da oferta de oportunidade de trabalho, aposentadoria, vínculos familiares sólidos, apoio social, educação e aprendizagem permanente, proteção contra a violência e maus - tratos, acesso a cuidado primário com assistência à saúde física e mental. Logo, a equipe de enfermagem recebe destaque quanto a essas medidas preventivas ao estimular estilos de vida saudável e participação ativa no cuidado da própria saúde ${ }^{(35)}$.

Os enfermeiros têm responsabilidades importantes na educação dos pacientes sobre os seus problemas de saúde, autocuidado e prevenção de danos. A orientação do autocuidado dos pacientes sobre a importância com a saúde torna o enfermeiro um componente-chave para a qualidade do cuidado $^{(36)}$. Entretanto, a competência técnica, intelectual e interpessoal é necessária para a coleta e interpretação dos dados dos pacientes, bem como o planejamento correto das intervenções e, consequentemente, os resultados esperados ${ }^{(37)}$.

Assim, a implantação de atividades que fortaleçam a promoção à saúde do idoso e minimizem os fatores de risco para a depressão como o desenvolvimento de ações de educação em saúde a fim de sensibilizá-los quanto a importância dos hábitos saudáveis de vida, incluindo a prática de atividade física e de atividades de lazer, que podem ocorrer em grupo, favorecendo ainda a interação social e ampliando a rede de apoio entre os idosos; podem ser realizadas por intermédio de profissionais de enfermagem ${ }^{(38)}$.

Embora tenham havido esforços consideráveis para projetar um estudo sólido e com resultados condizentes com a realidade da maioria da população idosa, houve limitação neste estudo. Os resultados obtidos estabelecem, na maior parte, uma relação mais associativa do que causal dos fatores de risco com o desenvolvimento da depressão. No entanto, apesar dessa limitação, este estudo identificou os fatores de risco relacionados ao desenvolvimento da depressão e fornece evidências sobre a saúde da população em estudo, suas 
vulnerabilidades e, pode ser utilizado para o desenvolvimento de intervenções que previnam a ocorrência da depressão na população idosa.

\section{CONCLUSÃO}

O estudo identificou que os fatores associados à depressão em idosos são o baixo nível de escolaridade, estado civil divorciado, solteiro ou viúvo, presença de mais de uma patologia crônica, ausência de atividades sociais, de lazer e de práticas de atividades físicas, dificuldades financeiras, residentes em áreas rurais e lares de idosos, morar sozinho, limitação funcional, suporte social precário, conflitos ou tempo limitado de convívio com a família, histórico de morbidades psicológicas e uso de mais dois medicamentos regularmente.

Ressalta-se que esses resultados podem subsidiar ações de saúde voltadas de modo particular aos idosos que apresentem os fatores de risco citados, agindo de forma preventiva e precoce à identificação da depressão.

Desta forma, destaca-se a importância de ampliar os estudos para investigar os preditores de depressão em idosos de maneira mais extensiva, dada à relevância e influência do reconhecimento destes fatores com a finalidade de promover intervenções precoces para esta população. Assim, é essencial investimentos e valorização de estratégias que visem capacitar os profissionais de saúde acerca dos sinais relacionados ao risco de depressão a serem explorados na população idosa.

Portanto, após identificar esses elementos, a promoção de ações assistenciais que minimizem os fatores de risco reconhecidos é fundamental e, além de zelar pela qualidade de vida da pessoa idosa, contribui para o processo de envelhecimento digno fisicamente e emocionalmente.

\section{REFERÊNCIAS}

1.Organização Mundial da Saúde. Depressão. [Internet] 2017. [acesso 18 novembro de 2020]. Disponível em: https://www.paho.org/pt/topicos/depressao

2. Cruwys T, Haslam SA, Dingle GA, Haslam C, Jetten J. Depression and social identity: and integrative review. Personality and Social Psychology Review. 2014; 18 (3): 215-38. Disponível em: https://journals.sagepub.com/doi/10.1177/108 8868314523839

3. Videbeck Sheila L. Enfermagem em saúde mental e psiquiatria. 5 ed. Porto Alegre: Artmed; 2012.

4. Rufino S, Leite RS, Freschi L, Venturelli VK, Oliveira ES, Filho DAMM. Aspectos gerais, sintomas e diagnóstico da depressão. 
Rev. Saúde em Foco. [Internet] 2018 [acesso em 20 novembro de 2020] 10 ed. Disponível em: https://portal.unisepe.com.br/unifia/wpcontent/uploads/sites/10001/2018/11/095_AS PECTOS-GERAIS-SINTOMAS-EDIAGN\%C3\%93STICO-DA-

DEPRESS\%C3\%83O.pdf

5. Gracioli J. Brasil vive surto de depressão e ansiedade. [Internet] 2018. [acesso em 19 novembro de 2020]. Disponível em: https://jornal.usp.br/atualidades/brasil-vivesurto-de-depressao-e-ansiedade/

6. Gandra A. Pesquisa revela aumento da ansiedade entre brasileiros na pandemia. [Internet] 2020. [acesso em 26 janeiro e 2021]. Disponível em: https://agenciabrasil.ebc.com.br/saude/noticia/ 2020-10/pesquisa-revela-aumento-daansiedade-entre-brasileiros-na-pandemia

7. Brasil, Instituto Brasileiro de Geografia e Estatística. Idosos indicam caminho para uma melhor idade. [Internet] 2019. [acesso em 19 novembro de 2020]. Disponível em: https://censo2021.ibge.gov.br/2012-agenciade-noticias/noticias/24036-idososindicamcaminhosparaumamelhoridade.html\#: 〜:text=Idosos \%20indicam\%20caminhos $\% 20 \mathrm{p}$ ara\%20uma\%20melhor\%20idade,Editoria\%3 A\%20Revista\%20Retratos\&text=De\%20acor do\%20com\%20a\%200rganiza\%C3\%A7\%C3 \%A3o,13\%25\%20da\%20popula\%C3\%A7\%C 3\%A3o\%20do\%20pa\%C3\%ADs
8. Fernandes I, Neves F, Guimarães P, Rolim KM, Albuquerque F, Andrade L et al. Perfil sociodemográfico da depressão em idosos no Brasil: revisão integrativa. Millenium. [Internet] 2020 [acesso em 20 novembro de 2020] 2(12):79-84. Disponível em: file://D:/Users/Windows\%2010/Downloads/ DialnetPerfilSociodemograficoDaDepressaoE mIdososNoBrasilR-7668055\%20(1).pdf

9. Sousa KA, Freitas FFQ, Castro AP, Oliveira CDB, Almeida AAB, Sousa KA. Prevalência de sintomas de depressão em idosos assistidos pela estratégia de saúde da família. REME Rev. Min. Enferm. [Internet] 2017 [acesso em 20 novembro de 2020] 21(2):82-93. Disponível em: http://www.dx.doi.org/10.5935/14152762.20170028

10. Brasil, Organização Pan-Americana de Saúde. Folha informativa- Envelhecimento e Saúde. [Internet] 2018. [acesso em 25 janeiro de 2021]. Disponível em: https://www.paho.org/bra/index.php?option=c om_content $\&$ view $=$ article $\& i d=5661:$ folhainformativa-envelhecimento-esaude $\&$ Itemid $=820$.

11. Medeiros GLF, Toledo MA, Sousa MNA. Depressão em Idosos: Implicações sociais e outras intercorrências. Id online Rev. Multi. Psic. [Internet] 2020 [acesso em 20 novembro de 2020] 14(53): 474-83. Disponível em: 10.14295/idonline.v14i53.2849 
12. Souza MT, Silva MD; Carvalho R. Revisão integrativa: o que é e como fazer. Revista Einstein São Paulo. [Internet] 2020 [acesso em 20 dezembro de 2020] 1(8):102-6. Disponível em: https://www.scielo.br/scielo.php?pid=S1679$\underline{45082010000100102 \& \text { script }=\text { sci_abstract } \& \text { tl }}$ $\underline{\mathrm{ng}=\mathrm{pt}}$

13. Galvão CM. Níveis de Evidência. Acta Paul Enferm. [Internet] 2006 [acesso em 05 de de janeiro de 2021] 19 (2). Disponível em:

https://www.scielo.br/scielo.php?script=sci_a rttext\&pid=S0103-21002006000200001

14. Stanetić K, Petrović V, Stanetić B, Kević1V, Stanetić M, Matović J, et al. Screening of undiagnosed depression among elderly primary care patients: a crosssectional study from the Republic of Srpska, Bosnia and Herzegovina. Med Glas (Zenica). [Internet] 2020 [acesso em 05 de de janeiro de 2021] 1(17): 200-5. Disponível em: https://pubmed.ncbi.nlm.nih.gov/31994858/

15. Buvneshkumar M, John KR, Logaraj M A. A study on prevalence of depression and associated risk factors among elderly in a rural block of Tamil Nadu. Indian Journal of Public Healt. [Internet] 2018 [acesso em 05 de de janeiro de 2021] 2(622):89-94. Disponível

em: https://www.ijph.in/article.asp?issn=0019557 $\underline{X} ;$ year $=2018 ;$ volume $=62 ;$ issue $=2 ;$ spage $=89 ; \mathrm{e}$ page $=94$;aulast $=$ Buvneshkumar
16. Kouvatsou k, Iliadou M, Kalatzi P, Evanthia E, Christos P, Maria K, et al. Depression Among Elderly Users of Open and Closed Care Facilities in a Rural Region of Greece: an Important Public Health Issue. Mater Sociomed. [Internet] 2020 [Acesso em 27 de dezembro de 2020]; 32(1): 35-40. Disponível

em: https://pubmed.ncbi.nlm.nih.gov/32410889/

17. Akila GV, Arvin BA, Isaac A. Comparative assessment of psychosocial status of elderly in urban and rural areas, Karnataka, India. Journal of Family Medicine and Primary Care. [Internet] 2019 [Acesso em 27 de dezembro de 2020] 8(9): 2870-76. Disponível em: https://www.ncbi.nlm.nih.gov/pmc/articles/P MC6820432/

18. Manandhar K, Risal A, Shrestha O, Manandhar N, Kunwar D, Koju1 R, et al. Prevalence of geriatric depression in the Kavre district, Nepal: Findings from a cross sectional community survey. BMC Psychiatry [Internet] 2019 [Acesso em 27 de dezembro de 2020] 19 (271): 2-9. Disponível em: https://bmcpsychiatry.biomedcentral.com/arti cles/10.1186/s12888-019-2258-5.

19. Zhong B, Xu YM, Xie WX, Liu XJ, Huang ZW. Depressive Symptoms in Elderly Chinese Primary Care Patients: Prevalence andSociodemographic and Clinical Correlates. Journal of Geriatric Psychiatry and Neurology [Internet] 2019 [Acesso em 27 
de dezembro de 2020]; 32(6) 312-8.

Disponível

em:

https://pubmed.ncbi.nlm.nih.gov/31480989/

20. Oliveira DV, Pivetta NRS, Oliveira GVN,

Silva DA, Júnior JRAN, Cavaglieri CR.

Fatores intervenientes nos indicativos de depressão em idosos usuários das unidades básicas de saúde de Maringá, Paraná, 2017*. Epidemiol. Serv. Saude, Brasília [Internet] 2019 [Acesso em 27 de dezembro de 2020]; 28(3):2018043. Disponível em: https://pesquisa.bvsalud.org/portal/resource/\% 20es/mdl-31800866

21. Lampert CDT, Ferreira VRT. Fatores Associados à Sintomatologia Depressiva em Idosos. Avaliação Psicológica [Internet] 2018 [Acesso em 28 de dezembro de 2020]; 17(2): 205-12.

Disponível

em:

http://pepsic.bvsalud.org/scielo.php?script=sc i_arttext\&pid=S167704712018000200007\&ln $\mathrm{g}=\mathrm{pt} \& \mathrm{nrm}=$ iso\&tlng=pt

22. Mendes-Chiloff CL, Lima MCP, Torres AR, Santos JLF, Duarte YO, Lebrão ML, et al. Sintomas depressivos em idosos do município de São Paulo, Brasil: prevalência e fatores associados (Estudo SABE). REV BRAS EPIDEMIOL [Internet] 2018 [Acesso em 28 de dezembro de 2020]; 21 (2): 180014. Disponível em: https://www.scielo.br/scielo.php?script=sci_a bstract\&pid=S1415790X2018000300411\&lng=pt\&nrm=iso
23. Rubiano EK, Frias DR, Abarca IO, Villanueva MV, Martíneza EB, Villalba MC, et al. Riesgo de depresiónen personas de 75 años o más, valoración geriátrica integral y factores de vulnerabilidade associados en Atención Primaria. Atención Primaria [Internet] 2015 [Acesso em 28 de dezembro de 2020]; 47 (10): 616-25. Disponível em: https://www.elsevier.es/es-revista-atencionprimaria-27

24. Zou C, Chen S, Shen J, Zheng X, Wang L, Guan L, et al. Prevalence and associated factors of depressive symptoms among elderly inpatients of a Chinese tertiary hospital. Clinical Interventions in Aging [Internet] 2018 [Acesso em 28 de dezembro de 2020]; 13: 1755-62. Disponível em: https://pubmed.ncbi.nlm.nih.gov/30271130/ 25. Nóbrega IRAP, Leal MCC, Marques APO, Vieira JCM. Fatores associados à depressão em idosos institucionalizados: revisão integrativa. Saúde em Debate [Internet] 2015 [acesso em 04 de janeiro de 2021]; 39: 536-50. Disponível em: https://www.scielosp.org/article/sdeb/2015.v3 9n105/536-550/

26. Schenker M, Costa DH. Avanços e desafios da atenção à saúde da população idosa com doenças crônicas na Atenção Primária à Saúde. Ciência \& Saúde Coletiva. [Internet] 2019 [acesso em 04 de janeiro de 2021] 24(4):1369-80. Disponível em: 
https://scielosp.org/pdf/csc/2019.v24n4/1369$1380 / \mathrm{pt}$

27. Guimarães L, Brito T, Pithon K, Jesus C, Souto C, Souza S, Santos T. Sintomas depressivos e fatores associados em idosos residentes em instituições de longe permanência. Ciênc. Saúde Coletiva. [Internet] 2019 [acesso em 05 de janeiro de 2021]; 24(9): 3275-82. Disponível em: https://www.scielosp.org/article/csc/2019.v24 n9/3275-3282/

28. Gutiérrez J, Moreno I, Aragón M. Characteristics and factors associated with depression in the elderly in Spain geom a gender perspective. Actas Esp Psiquiatr. [Internet] 2017 [acesso em 04 de janeiro de 2021]; 45(5): 185-200. Disponível em: https://pubmed.ncbi.nlm.nih.gov/29044444/

29. Koç Z, Saglam Z. Determination of the effects of daily life acrivities and self-care capacity on depression of the elderly in northern turkey. Acta Clin Croat. [Internet] 2019 [acesso em 05 de janeiro de 2021]; 58:516-22. Disponível em: https://hrcak.srce.hr/index.php?show=clanak $\underline{\text { \&id_clanak_jezik=334752 }}$

30. Almomani FM, Bani-issa W. The incidence of depression among residents of assisted living: prevalence and related risk factors. Clin Interv Aging [Internet] 2017 [acesso em 05 de de janeiro de 2021]; 12: 1645-53.

Disponível

em:
https://www.ncbi.nlm.nih.gov/pmc/articles/P $\underline{\mathrm{MC} 5640406 /}$

31. Lau YW, Vaingankar JA, Abdin E, Shafie S, Jeyagurunathan A, Zhang Y et al. Social support network typologies and their association with dementia and depression among older adults in Singapore: a crosssectional analysis. BMJ Open [Internet] 2019 [acesso em 05 de janeiro de 2021]; 9 (5): 025303. Disponível em: https://www.ncbi.nlm.nih.gov/pmc/articles/P MC6549623/.

32. Arrieta H, Rezola-Pardo C, Echeverria I, Iturburu M, Gil SM, Yanguas JJ. Physical activity and fitness are associated with verbal memory, quality of life and depression among nursing home residents: preliminary data of a randomized controlled trial. BMC Geriatr. [Internet] 2018 [acesso em 06 de janeiro de 2021]; 18:80. Disponível em: https://www.ncbi.nlm.nih.gov/pmc/articles/P MC5869769/.

33. Choudhary A, Pathak A, Manickam P, Purohit M, Rajasekhar TD, Dhoble P et al. Effect of Yoga versus Light Exercise to Improve Well-Being and Promote Healthy Aging among Older Adults in Central India: A Study Protocol for a Randomized Controlled Trial. Geriatrics (Basel) [Internet] 2019 [acesso em 06 de janeiro de 2021]; 4(4): 64. Disponível em: https://www.ncbi.nlm.nih.gov/pmc/articles/P MC6960920/. 
34. Chen KM, Chen MH, Lin MH, Fan JT, Lin HS, Li CH. Effects of Yoga on Sleep Quality and Depression in Elders in Assisted Living Facilities. Journal of Nursing Research [Internet] 2010 [acesso em 05 de janeiro de 2021]; 18(1): 53-61. Disponível em: https://journals.lww.com/jnr-

twna/Fulltext/2010/03000/Effects_of_Yoga_o n_Sleep_Quality_and_Depression_in.9.aspx

35. França CL, Murta SG. Prevenção e Promoção da Saúde Mental no Envelhecimento: Conceitos e Intervenções. PSICOLOGIA: CIÊNCIA E PROFISSÃO [Internet] 2014 [Acesso em 06 de janeiro de 2021]; 34(2): 318-29. Disponível em: https://www.scielo.br/scielo.php?script=sci_a $\underline{\text { rttext\&pid }=S 1414-98932014000200005}$

36. Oermann MH, Templin T. Important attributes of quality health care: consumer perspectives. J Nurs Scholarsh. [Internet]2000 [acesso em 11 de fevereiro de 2021]; 32(2):167-72. Disponível em: https://pubmed.ncbi.nlm.nih.gov/10887716/ 37. Lunney M. Helping nurses use NANDA, NOC and NIC. Novice to expert. J Nurs Adm. [Internet]2006 [acesso em 11 de fevereiro de 2021]; 36(3):118-25. Disponível em: https://pubmed.ncbi.nlm.nih.gov/16601513/

38. Ferreira PCS, Tavares DMST, Martins NPF, Rodrigues LR, Ferreira LA. Características sociodemográficas e hábitos de vida de idosos com e sem indicativo de depressão. Rev. Eletr. Enf. [Internet]. 2013
[Acesso em 06 de Janeiro de 2021]; 15(1):197-204. Disponível em: http://dx.doi.org/10.5216/ree.v15i1.16643.

Submissão: 2021-02-18

Aprovado: 2021-05-04 